\title{
Atrial Flutter in a Newborn: a Case Report
}

\author{
Andreea Paler Cerghit ${ }^{1}$, Amalia Făgărășan ${ }^{1,2}$, Iolanda Muntean ${ }^{1,2}$, Sorina Pasc ${ }^{1,2}$, Dorottya \\ Miklósi ${ }^{1}$, Liliana Gozar ${ }^{1,2}$, Rodica Togănel ${ }^{1,2}$ \\ 1 "George Emil Palade" University of Medicine, Pharmacy, Science and Technology, Târgu Mureș, Romania \\ ${ }^{2}$ Emergency Institute of Cardiovascular Diseases and Transplantation, Târgu Mureș, Romania
}

\section{ABSTRACT}

Introduction: The incidence of cardiac arrhythmia is approximately $1 \%$ in the neonatal period and $1-3 \%$ in late pregnancy. Atrial flutter $(\mathrm{AF})$, a rhythm disorder based on the mechanism of reentry, represents approximately $32 \%$ of all neonatal cardiac arrhythmias. In the majority of cases, the flutter is converted to sinus rhythm using antiarrhythmic drugs, transesophageal overdrive pacing, or synchronized electrical cardioversion. Case presentation: We present a case of a born on term, female infant who was noted to be tachycardic on the fetal monitor. Clinical examination revealed tachypnea and tachycardia with dysrhythmia, at a heart rate of $250 \mathrm{bpm}$. Electrocardiography showed AF with 2-3:1 atrioventricular conduction (atrial and ventricular rates were $350 \mathrm{bpm}$ and 250-275 bpm, respectively). Echocardiography revealed no relevant structural disease. Therapy with amiodarone and prophylactic anticoagulant was initiated. As no control of ventricular rate was obtained and a succession of three ventricular extrasystoles was noted on the surface ECG, propranolol therapy was initiated, without success. Therefore, synchronized electrical cardioversion was applied, with conversion to sinus rhythm at a heart rate of $136 \mathrm{bpm}$. The neonate was discharged in good condition. Conclusions: $\mathrm{AF}$ is one of the most common high ventricular rate arrhythmias during fetal age. Uncontrolled AF may precipitate heart failure, and prompt restoration to sinus rhythm may require electrical cardioversion in cases refractory to anti-arrhythmic drugs, in order to prevent possible complications.

Keywords: atrial flutter, neonates, antiarrhythmics, synchronized electrical cardioversion

\section{ARTICLE HISTORY}

Received: December 19, 2019

Accepted: July 6, 2020

\section{CORRESPONDENCE}

Rodica Togănel

Str. Gheorghe Marinescu nr. 38 540139 Târgu Mureș, Romania Tel: +40745652688

E-mail: rodicatoganel@yahoo.com

\section{INTRODUCTION}

The incidence of cardiac arrhythmia is approximately $1 \%$ in the neonatal period and $1-3 \%$ during the late phases of pregnancy. ${ }^{1}$ The most common arrhythmias in the neonatal age group are sinus tachycardia, atrioventricular reentrant tachycardia, and atrial flutter $(\mathrm{AF})$, which is seldom encountered outside the neonatal period. ${ }^{2}$
AF represents about $32 \%$ of all neonatal cardiac arrhythmias, and it is based on a reentry mechanism. ${ }^{3} \mathrm{AF}$ is usually diagnosed on the electrocardiogram (ECG) in the presence of typical saw-tooth flutter waves that are best seen in leads II, III, and aVF. Atrial rates vary between $280-450$ beats per minute (bpm), frequently accompanying a $2: 1$ atrioventricular conduction and a ventricular response ranging from 150 to $250 \mathrm{bpm} .{ }^{4}$ The arrythmia may 


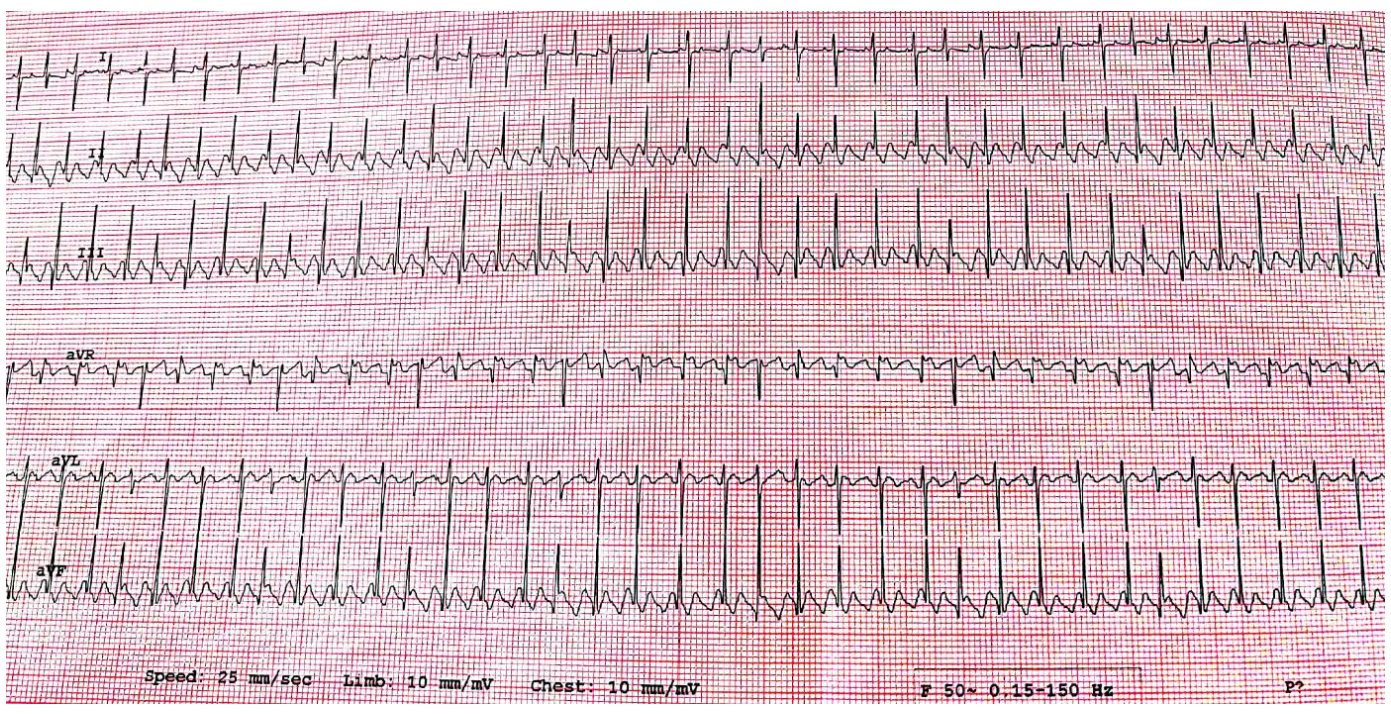

FIGURE 1. ECG showing typical saw-tooth flutter waves that are best seen in leads II, III, and aVF

be easily converted to sinus rhythm by cardioversion or by using transesophageal overdrive pacing. ${ }^{5}$

\section{CASE PRESENTATION}

We present the case of a born to term female infant, weighing 3,900 $\mathrm{g}$ at birth, from the third pregnancy of a 25-year-old mother. The pregnancy was not followed-up by a gynecologist. When the mother had been admitted to the hospital due to uterine contractions, the fetus was noted to be tachycardic during fetal monitoring. Therefore, a cesarean section was performed due to fetal dis- tress. Postnatally, the APGAR scores of the infant were 9 and 10 at 1 and 5 minutes, respectively. The neonate was still noted to present tachycardia, with heart rates of 196 bpm, and tachypnea, with a peripheral oxygen saturation of $98-99 \%$ and a blood pressure of $80 / 48 \mathrm{mmHg}$, and was referred to a clinic of pediatric cardiology.

On admission, the clinical examination revealed tachypnea (60/min) and tachycardia (250 bpm). The peripheral oxygen saturation was $99 \%$, and a systolic murmur (3/VI) was recorded at the superior left sternal border. The liver was enlarged at approximately $2 \mathrm{~cm}$ below the right costal margin. The ECG tracing demonstrated AF with 2-3:1

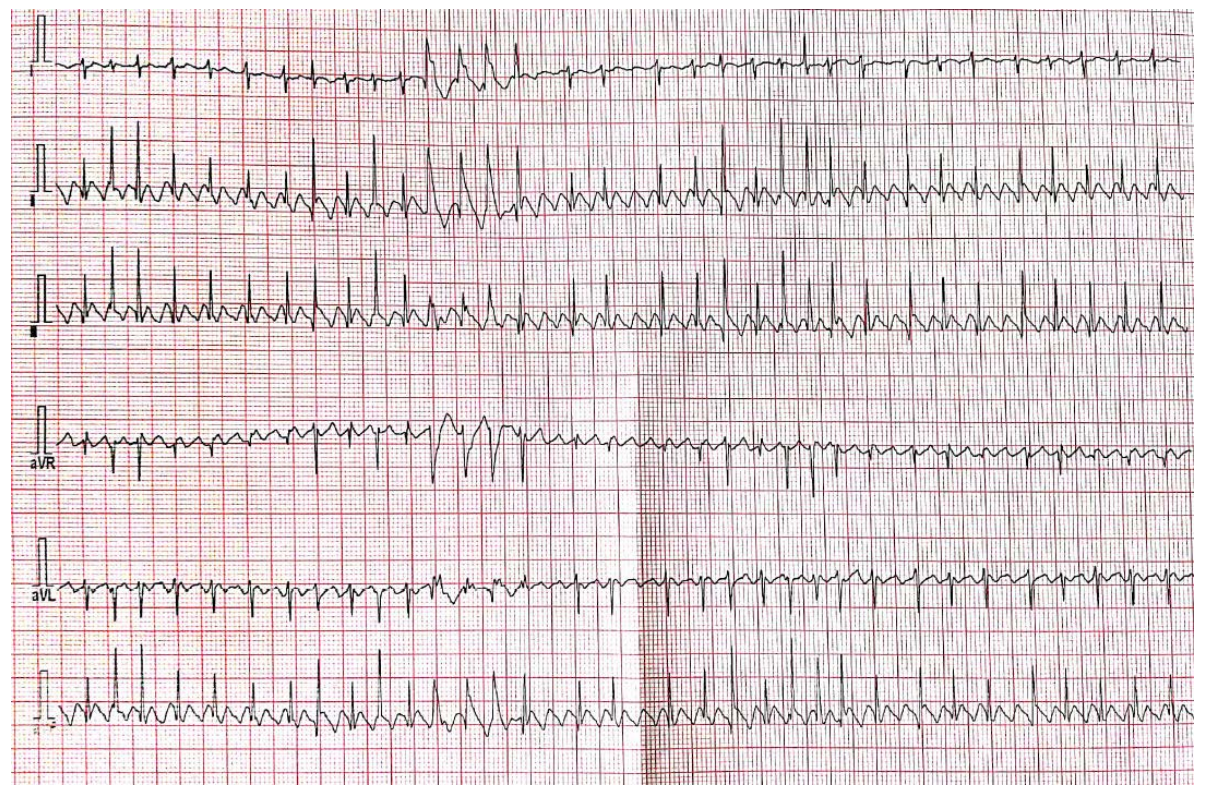

FIGURE 2. ECG showing atrial flutter and a series of three ventricular extrasystoles 


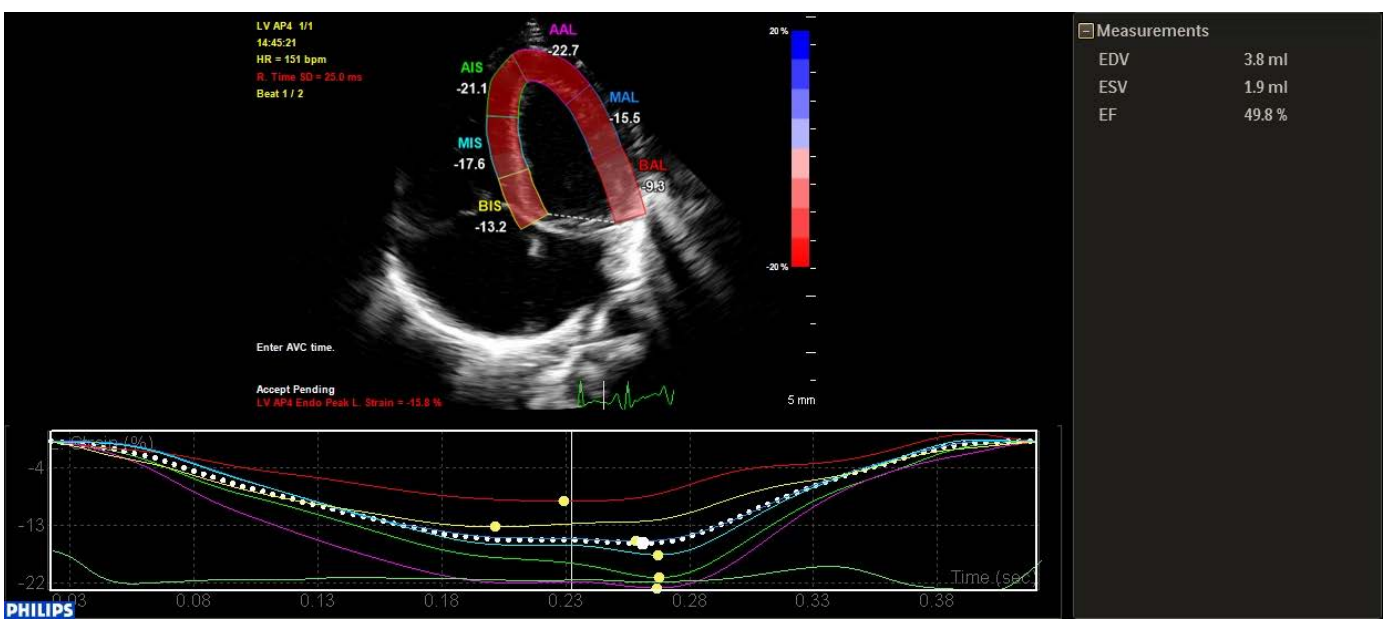

FIGURE 3. Speckle-tracking measurements of global longitudinal strain (white dotted line), calculated by averaging measurements of six segments in the apical four-chamber view

atrioventricular conduction, with an atrial rate of $350 \mathrm{bpm}$ and a ventricular rate of 250-275 bpm (Figure 1). Echocardiography revealed neonatal pulmonary hypertension in the absence of any structural disease, a small ostium secundum atrial septum defect with right-to-left shunt, dilated right atrium with spontaneous echo contrast, and diminished contractility with an ejection fraction (EF) of $38 \%$, without any evidence of intracavitary thrombus. Therefore, intravenous amiodarone was initiated (at a dosage of $5 \mathrm{mg} / \mathrm{kg}$ in slow infusion over $60 \mathrm{~min}$, followed by a maintenance dose of $5 \mu \mathrm{g} / \mathrm{kg} / \mathrm{min}$ ), in parallel with prophylactic anticoagulant therapy with enoxaparin. As after several hours no control of ventricular rate was obtained and a succession of three ventricular extrasystoles was noted on the ECG (Figure 2), propranolol therapy was associated. However, after 24 hours from the initiation of amiodarone and propranolol, the rhythm and rate control were still not achieved. Therefore, electrical external cardioversion was applied with 2 joule $/ \mathrm{kg}$, with conversion to sinus rhythm at a heart rate of $136 \mathrm{bpm}$. After cardioversion, the myocardial function was assessed using speckletracking echocardiography, which showed a good function of the left ventricle, with a global longitudinal strain peak of $-15.8 \%$ and the following segmental strain values: basal lateral $-9.3 \%$, mid lateral $-15.5 \%$, apical lateral $-22.7 \%$, basal septal $-13.2 \%$, mid septal $-17.6 \%$, apical septal $-21.1 \%$, EF $49.8 \%$ (Figure 3). Left ventricular global circumferential (C) peak strain was $-17.5 \%$, apical C strain $-22.5 \%$, mid C strain $-9.8 \%$, and basal C strain $-20.3 \%$ (Figure 4). The neonate was discharged in good condition, and therapy with propranolol and enoxaparin was ended. Amiodarone therapy was switched to oral maintenance

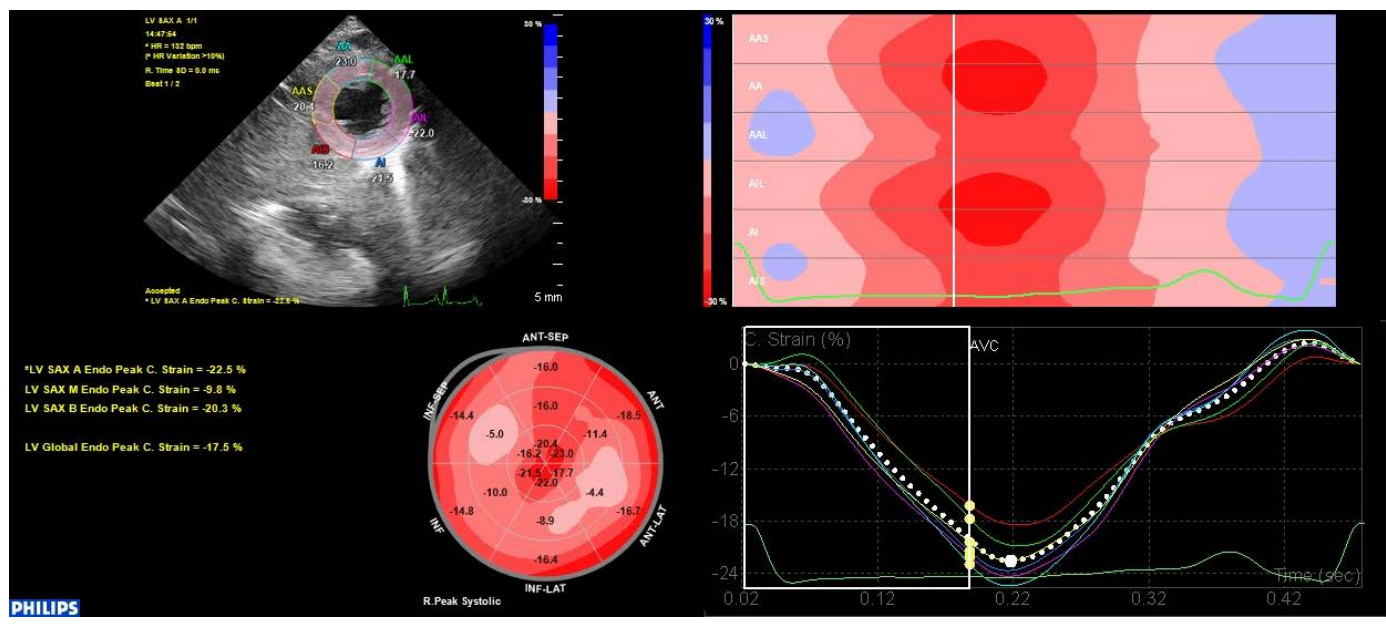

FIGURE 4. Speckle-tracking measurements of global circumferential strain (white dotted line), calculated by averaging measurements of six segments in parasternal short axis view 
doses and continued for prevention of recurrence, and the neonate was scheduled for a clinical follow-up.

The legal guardians/parents of the pediatric patient agreed to the publication of her data, and the institution where the patient had been admitted, approved the publication of the case.

\section{DISCUSSIONS}

Without the presence of a structural cardiac disease, atrial flutter is nearly always diagnosed during the last semester of pregnancy or in the early neonatal period. ${ }^{6}$ The gender distribution of $\mathrm{AF}$ seems to be equal. ${ }^{3} \mathrm{AF}$ is generally well tolerated, and association with fetal hydrops or death is rarely encountered. ${ }^{6}$ The majority of fetal rhythm disorders detected before week 36 of gestation can be treated by maternal administration of antiarrhythmic drugs such as digoxin or dexamethasone. Dexamethasone is recommended in case of severe hydrops fetalis, but its neurologic side effects should also be taken into consideration. ${ }^{7}$ In our case, the pregnancy was not followed by a physician, but it had been carried out by the mother up to week 39 without any events. Therefore, we assumed that AF presenting in the fetus had appeared during the third trimester or the peripartum period, and therefore maternal therapy was not initiated. Furthermore, the newborn did not present any associated structural heart disease.

Fetuses and neonates with AF or ectopic atrial tachycardia are more likely to present macrosomia or to be born from diabetic mothers, compared with the general population. ${ }^{8}$ However, neither macrosomia nor gestational diabetes was present in our case.

$\mathrm{AF}$ is an arrythmia based on a macro-reentry mechanism, present within the atrial wall, with a variable conduction to the ventricles, and does not include the atrioventricular node. ${ }^{3,9}$ As the cardiac output is directly influenced by the ventricular rate, a very rapid ventricular depolarization may lead to a sudden decrease in the stroke volume and thus to acute heart failure..$^{10}$ The rapid atrial contractions with secondary blood pooling can be responsible for thrombus formation and may lead to embolic events. The objectives of the emergency therapy is to either cease the tachyarrhythmia or, if rhythm control is not achieved, to lower the rapid ventricular rate, in order to restore a proper cardiac output. ${ }^{9}$ If AF persists, sinus rhythm can be restored by transesophageal overdrive pacing or synchronized electrical cardioversion..$^{5}$ In the presented case, the control of both rhythm and rate was not achieved after the initiation of continuous infusion of amiodarone associated with propranolol. Sinus rhythm restoration was only achieved with synchronized electrical cardioversion. Prophylactic anticoagulation therapy was initiated to prevent the occurrence of thromboembolic events, which may be favored by the enlarged right atrium, the diminished contractility, and the presence of the ostium secundum atrial septal defect with right-to-left shunt.

The recurrence of AF in neonates is rare, and generally, long-term treatment is not required. ${ }^{9}$ For prevention of recurrence, class I (quinidine) and class III (amiodarone) antiarrhythmic agents may be effective in some cases. ${ }^{10}$ We decided to continue oral amiodarone therapy for the next month, until reevaluation. Although neonatal arrhythmias have a relatively good prognosis, regular follow-up of patients is crucial in the prevention of possible complications. ${ }^{3}$

\section{CONCLUSIONS}

$\mathrm{AF}$ is one of the most common high ventricular rate rhythm disorders in neonates. The majority of cases are easily converted to sinus rhythm by transesophageal overdrive pacing or by synchronized electrical cardioversion. Uncontrolled atrial flutter may precipitate heart failure, and prompt restoration to sinus rhythm may require electrical cardioversion in cases refractory to anti-arrhythmic drugs, in order to prevent possible complications.

\section{CONFLICT OF INTEREST}

Nothing to declare.

\section{REFERENCES}

1. Badrawi N, Hegazy RA, Tokovic E, Lotfy W, Mahmoud F, Aly $\mathrm{H}$. Arrhythmia in the neonatal intensive care unit. Pediatr Cardiol. 2009;30:325-330. doi: 10.1007/s00246-008-9355-4.

2. Low S, Kiper C, Armstrong A, Cua CL. Echocardiographic diagnosis of atrial flutter in a neonate. Echocardiography. 2018;35:1439-1441. doi: 10.1111/echo.14111.

3. Yllmaz-Semerci S, Bornaun H, Kurnaz D, et al. Neonatal atrial flutter: Three cases and review of the literature. Turk J Pediatr. 2018;60:306-309. doi: 10.24953/turkjped.2018.03.011.

4. Luewan S, Sittiwangkul R, Srisupundit K, Tongsong T. Perinatal treatment of refractory atrial flutter with hydrops fetalis: a case report. J Med Assoc Thai. 2011;94:878-881.

5. Moller JH, Davachi F, Anderson RC. Atrial flutter in infancy. J Pediatr. 1969;75:643-651. doi: 10.1016/s00223476(69)80461-0.

6. Jaeggi ET, Carvalho JS, De Groot E, et al. Comparison of transplacental treatment of fetal supraventricular tachyarrhythmias with digoxin, flecainide, and sotalol: results of a nonrandomized multicenter study. Circulation. 2011;124:1747-1754. doi:10.1161/CIRCULATIONAHA.111.026120. 
7. Sacks JH, Samai C, Gomez K, Kanaan U. Maternal antibodyassociated fetal second-degree heart block and atrial flutter: case report and review. Pediatr Cardiol. 2013;34:2040-2043. doi: 10.1007/s00246-012-0588-x.

8. Pike JI, Krishnan A, Kaltman J, Donofrio MT. Fetal and neonatal atrial arrhythmias: an association with maternal diabetes and neonatal macrosomia. Prenat Diagn. 2013;33:1152-1157. doi: 10.1002/pd.4210.

9. Jaeggi E, Öhman A. Fetal and Neonatal Arrhythmias. Clin Perinatol. 2016;43:99-112. doi: 10.1016/j.clp.2015.11.007.

10. Park Myung K. Park's the Pediatric Cardiology Handbook: Cardiac Arrithmias. 5th ed Elsevier, 2016; p. 269-294. 